\title{
Performance architecturale: una aproximación al concepto de dérive en la arquitectura de Rem Koolhaas
}

Performance architecturale: an approach to the concept of dérive in the architecture of Rem Koolhaas

Javier Arias Madero

Universidad de Valladolid, España

jarias@arq.uva.es

https://orcid.org/0000-0003-4090-4533 


\title{
Resumen
}

El movimiento es uno de los temas fundamentales de investigación creativa contemporánea. Comenzando por las vanguardias de las primeras décadas del siglo XX: futuristas, dadaístas, y después los surrealistas, todos hicieron del estudio del movimiento del sujeto en su contexto uno de sus principales campos de experimentación. Décadas después, letristas y situacionistas, reciclan la investigación precedente aumentando la connotación radical del deambular como liberación de la rutina y del orden establecido, abriendo futuras nuevas vías creativas de amplio espectro en disciplinas como el arte de acción, la performance, el teatro, la danza, el cine, pero también de modo relevante en el diseño urbano y en la arquitectura. Uno de los arquitectos que mejor recoge el testigo de toda esta experiencia de investigación con el movimiento y que consigue trasladar estos suculentos hallazgos al recorrido urbano y arquitectónico es Rem Koolhaas. Influido de modo importante en el inicio de su carrera por todas estas corrientes plásticas previas, la obra de Koolhaas se caracterizará por innovar de modo constante en el territorio del desplazamiento del individuo, transformándolo en una acción artística al margen del propio edificio. Una verdadera Performance Architecturale. Koolhaas reivindica la dèrive baudeleriana e instrumentaliza el deambular errático por la ciudad en gran parte de sus proyectos. No sólo lo hace en aquellos de carácter territorial. Sino que también experimenta con deambulatoria urbana dentro de los edificios, en coherencia una vez más, con su propuesta de una Arquitectura Metropolitana.

Palabras clave: Koolhaas; Baudelaire; deriva; recorrido; performance

\begin{abstract}
Movement is a major theme in contemporary creative research. It began with the avant-garde movements of the first decades of the twentieth century: Futurists, Dadaists, and later the Surrealists, all made movement in its context one of their main subjects of experimentation. Decades later, lettrists and situationists recycled previous research and reinforced the radical connotation of wandering as an act of liberation from routine and the established order. They paved the way towards new creative forms in a broad spectrum of disciplines such as action art, performance, theatre, dance, cinema, but also notably in urban design and architecture. One of the architects who best exploits all this research and past experience with movement and who manages to transfer these delectable gems to the urban and architectural domain is Rem Koolhaas. Decidedly influenced at the beginning of his career by all these plastic currents, Koolhaas's work would be characterised by constant innovations in the field of an individual's moving, transforming it into an artistic action outside the building itself. A true Performance Architecturale. Koolhaas vindicates the Baudelerian dérive and instrumentalises erratic city wandering in many of his projects. His contexts are not only territorial. Koolhaas also experiments with urban wandering within buildings, in coherence once again, with his proposal of Metropolitan Architecture.
\end{abstract}

Key words: Koolhaas; Baudelaire; drift; pathway; performance

\section{Para citar este artículo / To cite this article:}

ARIAS, J. Performance architecturale: una aproximación al concepto de dèrive en la arquitectura de Rem Koolhaas. En: [i2] Investigación e Innovación en Arquitectura y Territorio [en línea]. 2022, Vol.10, Núm. 1. ISSN: 2341-0515. https://doi.org/10.14198/12.19430

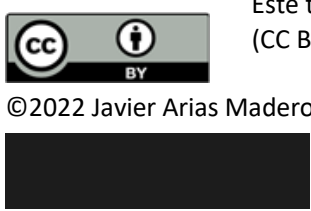

Este trabajo se publica bajo una licencia de Creative Commons Reconocimiento 4.0 Internaciona (CC BY 4.0): https://creativecommons.org/licenses/by/4.0/deed.es_ES 


\section{HIPÓTESIS}

Resulta sorprendente, cuanto menos, que el proyecto fin de carrera de un estudiante de arquitectura, que habitualmente consiste en proponer una solución arquitectónica a unas condiciones de partida, se inspire en un poema. Sin embargo, para aquel joven holandés la heterodoxia instrumental era ya una razón de ser y de producir. Rem Koolhaas, en 1972 estaba llamado a convertirse en un arquitecto disidente y a cuestionar el modus operandi del proyecto arquitectónico, así como a poner en crisis cualquier premisa dada. Aquel poema, extraído de las Flores del Mal de Charles Baudelaire formaba parte de su imaginario personal, cuyo interés se centraba en variopintas cuestiones no muy de moda entre los arquitectos de los años 70 del pasado siglo, como el surrealismo, el neorrealismo italiano, la internacional situacionista o les poètes maudits. La variopinta formación de Koolhaas, que le llevó a elegir la arquitectura como una vocación tardía tras sus experiencias como cineasta o periodista, forjaron una nutrida y divergente base epistemológica que no ha cesado de alimentar su producción crítica y arquitectónica durante todos estos años de profesión como arquitecto.

El objetivo del presente texto es demostrar que uno de los argumentos más robustos que justifica el nombre de la oficina de Koolhaas: Oficina para una Arquitectura Metropolitana es el modo de recorrer sus edificios. OMA no significa únicamente una arquitectura para la metrópolis, sino una arquitectura concebida internamente a partir de mecanismos metropolitanos. Y así, a aquellos postulados de la nueva arquitectura expresados en Delirious New York como el automonumento, la lobotomía y el cisma, (Koolhaas, 1978) nosotros propondremos añadir la deriva, en el sentido que veremos a continuación de recorrido urbano errático insertado en el edificio como componente sine qua non. Intentaremos demostrar, además, cómo la singular forma de concebir el recorrido en los edificios por Koolhaas, esta performance architecturale, entronca directamente con toda la experiencia de deambulatoria, de inspiración baudeleriana desarrollada por algunas de las principales corrientes creativas del siglo XX y que llega a nuestros días (Wiley, 2010).

\section{EXODUS: DEAMBULACIÓN Y PLACER}

La influencia de Baudelaire en la obra de Koolhaas es siempre tangible: su concepción apasionada de la metrópolis, la visión hedonista del mundo, la comunión entre lo divino y lo diabólico, y la apología del flâneur: el vagabundo que, rebelándose contra el orden establecido, pasa el tiempo recorriendo las calles sin rumbo conocido (Gargiani, 2008).

"El paseante solitario y pensativo saca una embriaguez particular ante esta universal comunión. El que fácilmente se desposa con la muchedumbre conoce placeres febriles, del que estarán eternamente privados el egoísta, cerrado como un cofre, y el perezoso, interno como un molusco." (Baudelaire, 2003: 35)

En esta idea de deriva del clochard por la ciudad, azarosa e imprevista; en ese caminar automático, encontraremos gran parte de la esencia de los recorridos en los edificios de OMA del que Exodus constituye un gran ejercicio teórico preliminar. 
El planteamiento de este proyecto, ganador del concurso internacional La città come ambiente significante organizado por la revista Casabella en 1972 y publicado al año siguiente en su número 378 , se inspiraba ya profundamente en el concepto de flânerie baudeleriana. Exodus inserta en el corazón de Londres dos muros paralelos separados unos ochocientos metros entre sí. En su interior crece una nueva ciudad, la Mitad Buena. El desarrollo longitudinal entre dichos muros genera plazas cuadradas independientes y cada una de ellas es una pieza del engranaje del funcionamiento social de la propuesta. El interior de la banda arrasa por completo con el tejido urbano del centro de Londres imponiendo su propia realidad. La disposición de las distintas zonas no tiene sentido funcional alguno desde un planteamiento racional urbano y esto implica que la vida en la franja precisa de una serie constante de deambulaciones erráticas, una deriva cotidiana, - fig.1- que incita a sus habitantes a recorrer todas las zonas mediante un continuo fluir de circulaciones peatonales que aseguren el intercambio personal y la interacción con la nueva ciudad.

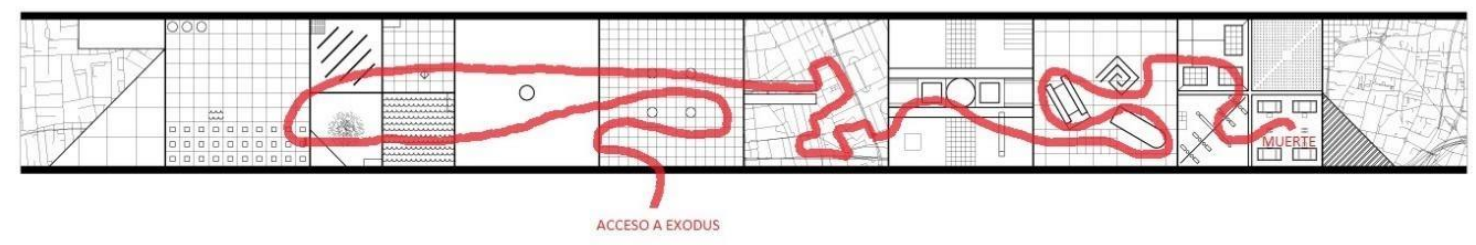

FIG. 1. Esquema del autor sobre los flujos de movimiento de los habitantes de Exodus en función de la descripción de Koolhaas de la vida en la franja recorriendo las distintas zonas del proyecto desde el ingreso en la ciudad hasta la muerte del individuo. Fuente: elaboración propia.

Koolhaas termina el relato de la memoria del proyecto remarcando su carácter de propuesta de intervención moral. Un modelo urbano que atesora un mensaje de ruptura, rebelión y de loa a este paraíso surrealista "el surrealismo es un paraíso harto artificial, y la afición a este paraíso deriva del estudio de Baudelaire" (Breton, 1969: 57). Como conclusión, el arquitecto holandés incluye un extracto de Sueño parisién, perteneciente a la aludida Las Flores del Mal de Baudelaire, que sintoniza perfectamente con la impactante experiencia del paseo por este paisaje paradisiaco de Exodus.

"Arquitecto de mis hechizos,

Yo hacía, a mi capricho,

un túnel de pedrerías

Para pasar un océano domado;

Y todo, aún el color negro,

Parecía límpido, claro, irisado;

El líquido engarzaba su gloria

En el rayo cristalizado.

¡Ningún astro, por lo demás, ningún rastro

De sol, siquiera en la parte baja del cielo, 
Para iluminar estos prodigios,

Que brillaban con su propio fuego!“(García, 2014: 311)

A partir de este minuto cero de la producción arquitectónica de Koolhaas, podemos aseverar que el modo de recorrer las propuestas urbanas y los edificios del arquitecto nunca ha dejado de ser uno de los aspectos fundamentales (Mosquera, 2016). No obstante, resulta complicado definir una característica común al planteamiento del recorrido en la dilatada y heterodoxa obra del holandés. Podríamos situar el arco conceptual de estas circulaciones entre lo que definiríamos como un deambular urbano errático y un recorrido iniciático irracional. Sí que hay algo siempre en común que podemos concluir: las circulaciones no son casi nunca fáciles: Koolhaas nos somete a circular, a la necesaria aprehensión de una importante densidad de acontecimientos, transformando nuestro recorrido en una experiencia situada por encima de lo que es estrictamente el objetivo principal del recorrido, apartándose en ocasiones de los tradicionales requisitos de funcionalidad y economía de trayecto.

Es claro, en este sentido, que hay puntos de conexión entre la Promenade Architecturale corbuseriana y la circulación Koolhaasiana (del Valle, 2006), en cuanto a que el recorrido forma parte intrínseca del hecho arquitectónico. Le Corbusier, sin embargo, plantea una deambulación racional en busca de una apropiación del espacio por parte del usuario, proponiendo un recorrido continuo y suave en el que prolifera el uso de rampas para salvar desniveles, y que permite la contemplación sin interrupciones visuales. Todo lo contrario de una circulación sincopada y nerviosa (Quetglas, 1980).

La promenade de Koolhaas, la trajectory, como él mismo la denomina (Gargiani, 2008), rompe deliberadamente la continuidad sensorial del recorrido arquitectónico corbuseriano: la rampa también existe y el plano inclinado o alabeado, pero también la escalera mecánica y el ascensor. Este mecanismo busca el impacto y la sorpresa en el recorrido. Un camino entre prodigios, como describiera el poeta francés en el anterior texto.

\section{DEAMBULACIÓN Y VANGUARDIA. Breve historia del itinerario como sublimación}

El 14 de abril de 1921, en París, tuvo lugar una experiencia que a la postre constituiría un hecho capital para el devenir del arte contemporáneo del siglo XX. Ese día, los dadaístas inauguraron la Grande Saison Dada, en la que se incluyeron unos paseos erráticos por zonas periféricas de la ciudad sin objetivo concreto más que experimentar sensaciones, encuentros o desencuentros inesperados. Con esta acción, se produce no solo un descarrilamiento del arte de los soportes gráficos tradicionales, sino también de las salas de espectáculos, comenzando una serie de actividades vinculadas a la deambulación y a la deriva; una forma de anti-arte que llegará hasta nuestros días, de paseantes en busca de lo que Walter Benjamin denominó la iluminación profana.

“...el que pasea, el que callejea son tipos iluminados igual que el consumidor de opio, el soñador, el ebrio. Y, sin embargo, son profanos. Para no hablar de esa droga terrible, nosotros mismos, que tomamos en soledad". (Benjamin, 1980: 41) 
El surrealismo adoptará rápidamente estos paseos erráticos por la ciudad y el campo como un experimento con lo irracional. André Breton, que ya participaba en las Saisons Dadá, considera este deambular, conversando y caminando durante días, como una exploración hasta los límites entre la vida consciente y la vida soñada (Careri, 2015). No es casualidad que a la vuelta de una de ellas escriba Poisson Soluble, que más tarde se convertiría en el primer manifiesto surrealista. Las deambulaciones surrealistas, aquellos recorridos interminables por la periferia de París, serán durante los primeros años de existencia del grupo, ingrediente indispensable su sistema creativo global (Careri, 2015).

En realidad, en estos paseos surrealistas los participantes no hacen otra cosa más que cambiar el papel por el espacio tridimensional de la ciudad, y el lápiz por su propio cuerpo, o lo que es lo mismo, la experimentación espacial de la escritura y el dibujo automático, técnicas pioneras surrealistas de incitación del inconsciente que tanto interesaron a Koolhaas. La deambulación será como una terapia de suspensión de las defensas: la ciudad como lienzo, pero también la ciudad como diván. En este sentido, la vinculación de Koolhaas con los procedimientos automáticos y en particular con los experimentos de deambulatoria, confirma que el ampliamente estudiado interés del arquitecto holandés por el surrealismo radica en el ámbito del procedimiento por encima de otras consideraciones y así lo expresará: "He estado siempre interesado por el surrealismo, pero más por su poder analítico que por su exploración del subconsciente o por su estética" (Arias, 2016: 91)

Uno de los grupos herederos de la deambulatoria surrealista, y del que Koolhaas tenía un conocimiento de primera mano (Basar, 2008), retoma toda esta experiencia en una nueva propuesta creativa que tendrá que ver mucho con el concepto de trajectory koolhaasiana. Nos referimos al situacionismo y al concepto de dèrive, propuesto por Guy Debord treinta años más tarde de los paseos erráticos del grupo de Breton. La deriva situacionista ahonda en la afectación psicológica del paseante al deambular, añadiendo al azar surrealista otros ingredientes como el deseo, la emoción o la curiosidad (Careri, 2015). La ciudad entendida como un texto a disposición del paseante. Un texto no solo para ser leído, sino para ser escrito (Yarimbaş, 2018).

"Una o varias personas que se entregan a la deriva renuncian durante un tiempo más o menos largo a las motivaciones normales para desplazarse o actuar en sus relaciones, trabajos y entretenimientos para dejarse llevar por las solicitaciones del terreno y por los encuentros que a él corresponden. La parte aleatoria es menos determinante de lo que se cree: desde el punto de vista de la deriva, existe en las ciudades un relieve psicogeográfico, con corrientes constantes, puntos fijos y remolinos que hacen difícil el acceso o la salida de ciertas zonas." (Debord, 1999: 50) 
Es clara la influencia en Koolhaas del situacionismo tanto en la figura de Debord como en la de Constant Nieuwenhuys (Zaera, 1990), al que el arquitecto llega incluso a entrevistar y sobre el que escribe en su etapa de periodista. Constant en el proyecto de Nueva Babilonia, de gran influencia en Exodus, transforma la deambulación situacionista en arquitectura y en ciudad. Continúa desarrollando las premisas de Debord aportando la idea del homo ludens: el hombre cuya vida está entregada totalmente a la creatividad y al juego. El homo ludens habita una ciudad de crecimiento aleatorio, automático y laberíntica, en la que los propios habitantes son los constructores - fig.2-. Constant para definir su circulación por Nueva Babilonia utiliza el término desorientación y laberinto dinámico, precedente claro del concepto de la trajectory de Koolhaas (Ducatez, 2005).

"Si en la sociedad utilitarista se busca por todos los medios una organización óptima del espacio, como garantía de eficacia y ahorro de tiempo, en Nueva Babilonia se otorga más importancia a la desorientación, que favorece la aventura, el juego, el cambio creativo" (Constant, 2011: 44).

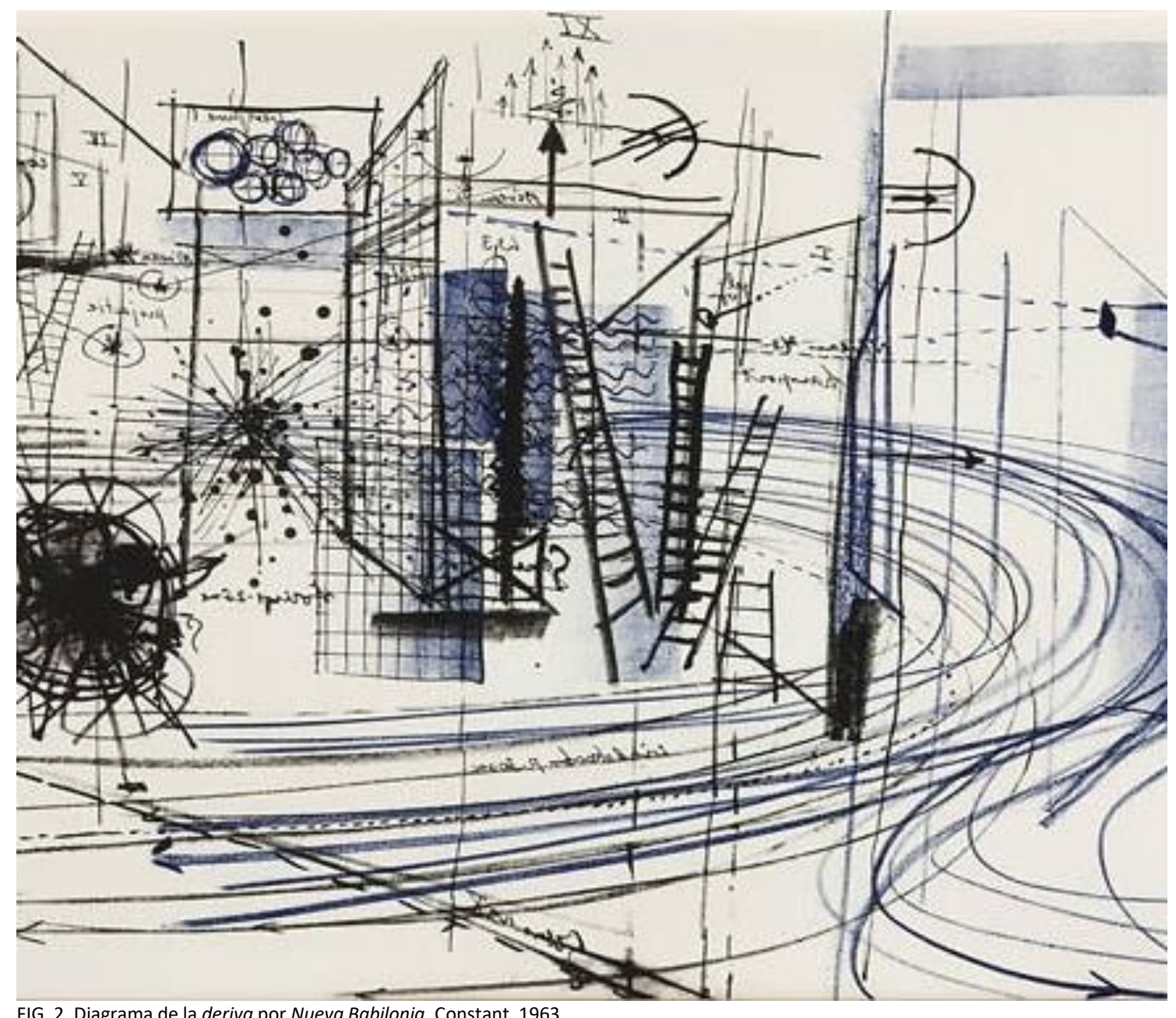

FIG. 2. Diagrama de la deriva por Nueva Babilonia. Constant, 1963. 
La trajectory de OMA es heredera de toda esta experiencia deambulatoria previa. Participa, como hemos visto, de aspectos comunes a la promenade architecturale corbuseriana, incluso al raumplan loosiano (Cortés, 2006), pero fundamentalmente instrumentaliza toda la carga irracional, automática y lúdica de la deambulatoria urbana surrealista y situacionista.

En este sentido, podemos diferenciar dos tipos de trajectory en las propuestas de OMA: la primera, aquella que persigue la libertad absoluta del individuo recorriendo un espacio, relacionada directamente con los conceptos anteriormente explicados del deambular surrealista y flânerie, y la segunda, otra línea de proyectos en la que el arquitecto holandés es el verdadero flâneur, siendo él quien concibe el laberinto dinámico en el edificio, definiéndolo y materializándolo arquitectónicamente de modo que el visitante se convierte en un fiel interprete del deambular circulatorio preestablecido por el creador de la obra.

\section{FLÂNERIE. Recorrido y libertad.}

Resulta difícil encontrar proyectos de cierta escala dentro de la dilatada obra de OMA en los cuales el recorrido no suponga el elemento fundamental vertebrador del mismo. Incluso en sus inicios, podemos referirnos a los grandes proyectos urbanos de los años 80 del pasado siglo, como las propuestas para Bijlmermeer, Melun-Sénart y Lille, o la influyente propuesta para el parisino Parque de la Villete, inspirado, entre otras cosas, en el movimiento mecánico del ascensor como recorrido estructurante del proyecto. Pero pronto Koolhaas empieza a utilizar recorridos urbanos como herramienta proyectual de propuestas puramente arquitectónicas, como la inserción de calles elevadas de las propuestas residenciales de IJ-Plein en Amsterdam, la de Boompjes en Roterdam o la del Check Point Charly berlinés (Mosquera, 2016), y pronto también comienza a distorsionar ese recorrido urbano interno hacia la experiencia singular $y$ sorprendente que supone el paseo errático.

Uno de los primeros proyectos de OMA donde se plasma de mejor modo el concepto de deambulatoria libre es el Centro de Congresos de Agadir en Marruecos, propuesta no construida de 1990. Se trata de un centro de convenciones con un teatro, un museo y un hotel. En esta ocasión OMA concibe un prisma cuadrangular de 140 metros de lado que secciona en dos, introduciendo un vacío ondulado como una prolongación del paisaje de las dunas de la playa donde se encuentra el complejo - fig.3-. 


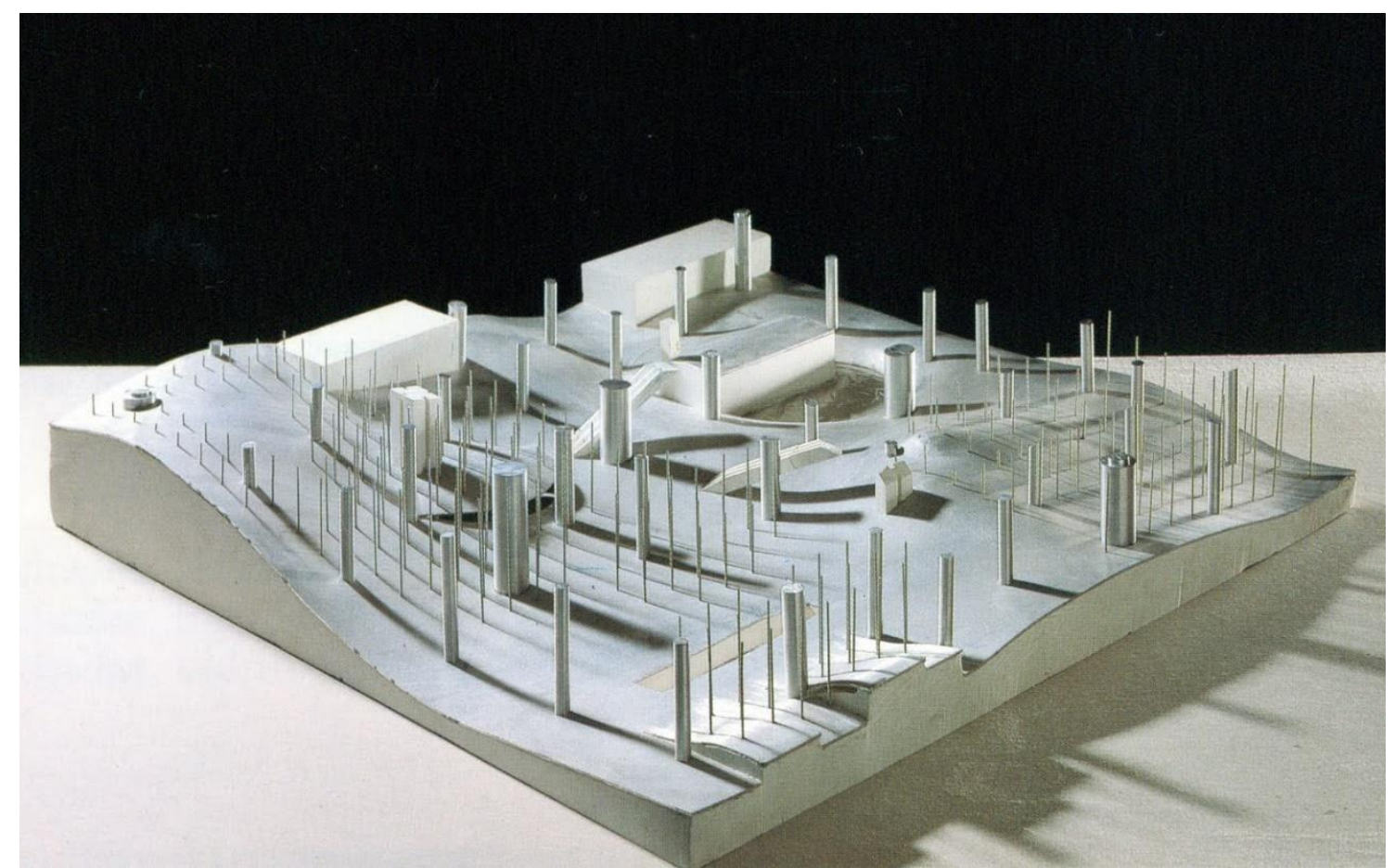

FIG. 3. OMA. Propuesta para el Centro de Congresos de Agadir, Marruecos. 1990. El prisma seccionado mostrando el nivel central, el vacío urbano de las dunas.

La parte fundamental del proyecto es esta superficie abierta que Koolhaas denomina la plaza urbana. En este espacio es donde se prevé un deambular incierto que promueva el encuentro interpersonal, "El paisaje que se genera con sus bóvedas cóncavas y convexas, con el bosque de columnas, sus rayos de luz, es una interpretación moderna del espacio islámico: Islam después de la relatividad de Einstein" (OMA, 2021). Las inclinaciones del suelo, sus colinas y valles, propiciarán los recorridos involuntarios de los usuarios, como en una cubierta de un barco que se mueve -fig.4-. La estructura soporte además complejiza estas derivas mediante la disposición de una colección aparentemente también automática de pilares de distintas proporciones y secciones.

Otro de los trabajos que mejor refleja el deambular automático y libre en las propuestas de OMA es el proyecto no construido de dos bibliotecas en la universidad de Jussieu, en París, en 1992. Como una perversión del modelo domino del Estilo Internacional, Koolhaas concibe un gran volumen prismático cuyos forjados, en distintos niveles, se pliegan y se cortan generando puntos de conexión inesperados. 


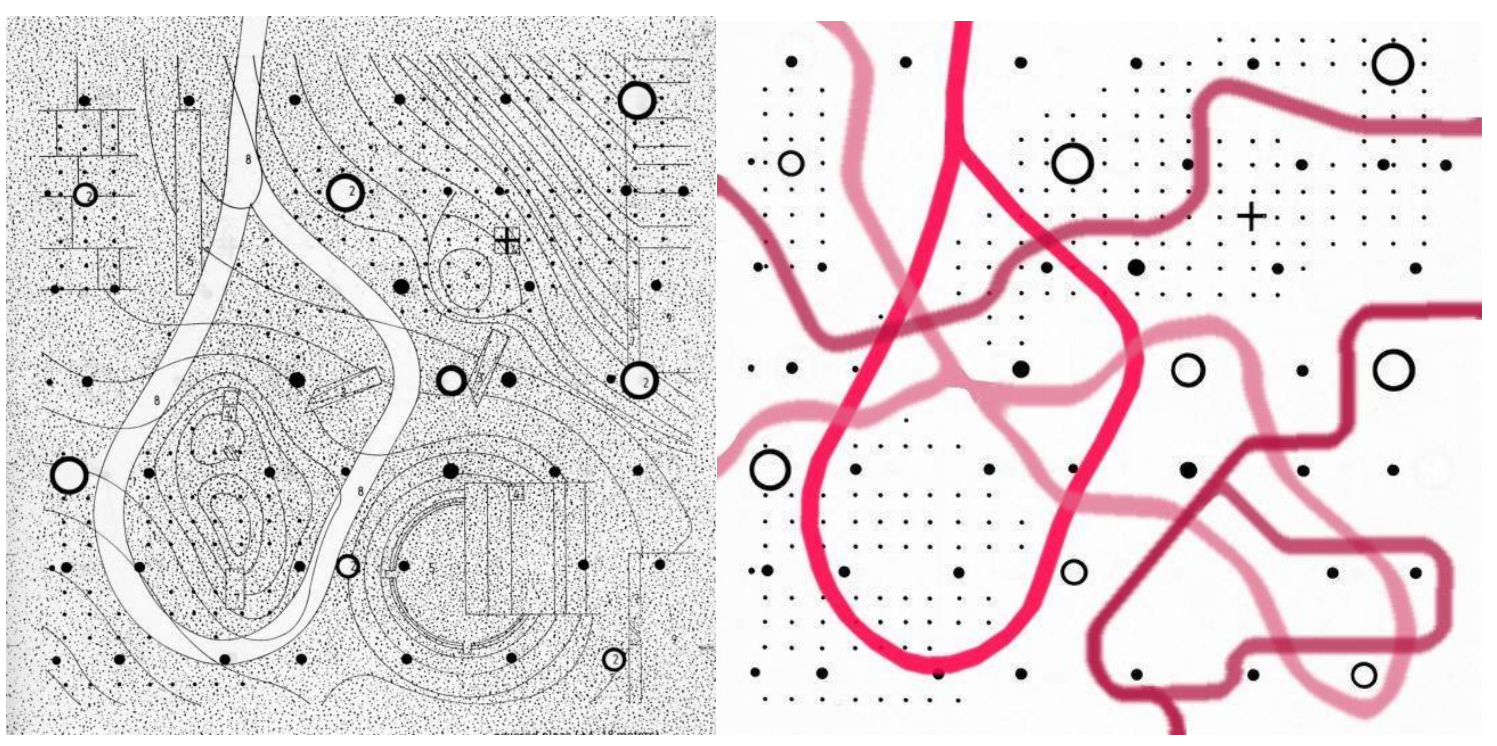

FIG. 4. Centro de Congresos de Agadir. Planta del nivel central y Esquema del autor de hipótesis de deambulaciones automáticas.

La circulación en el edificio es el principal elemento condicionante. De hecho, la idea inicial de la intervención se plantea como una solución a los problemas del tránsito peatonal en el complejo universitario. Koolhaas propone un edificio que condense en sí mismo la circulación como oposición a la dispersión en el gran atrio de la cota cero del complejo universitario, cuyos recorridos "agotan psicológicamente por anticipado cualquier intento de habitarlo" (Koolhaas, 1996: 124). El edificio se plantea como la solución para la red de las circulaciones peatonales en Jussieu en una operación conceptual de doblado múltiple de la superficie de la cubierta del podio, tal y como se plegaría una sábana que se guarda. - fig.5-

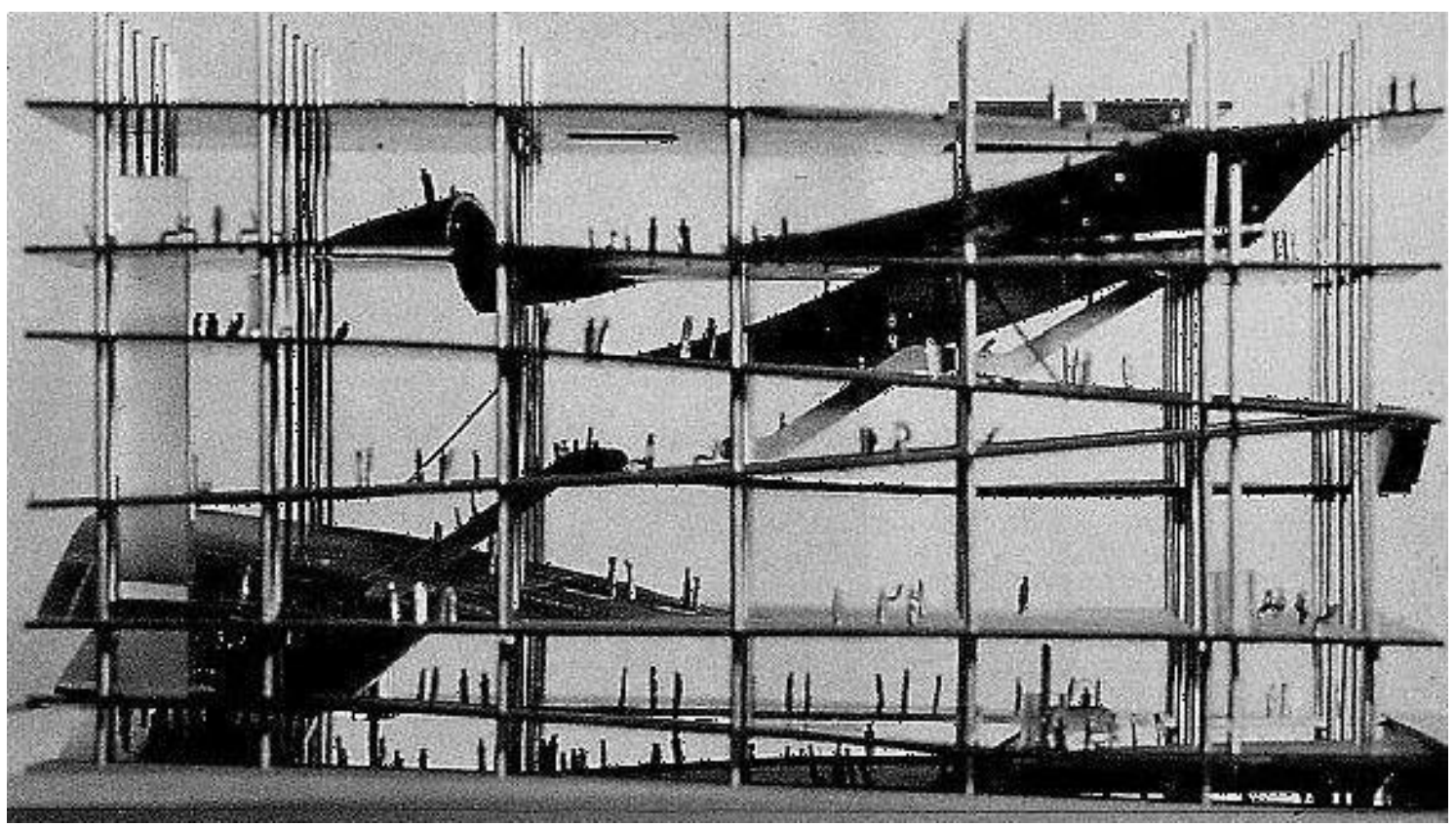

FIG. 5. OMA. Proyecto para dos bibliotecas en Jussieu, Francia. 1992. Maqueta donde se aprecia el carácter ausente del edificio dando protagonismo a las posibles deambulaciones por el mismo. 
Koolhaas vincula en su publicación $S, M, L, X L$ (Koolhaas, 1997) esta idea del proyecto al trabajo de Lygia Clark, y a sus performances psicoanalíticas. El arquitecto holandés relaciona el proceso del plegado del suelo del edificio de Jussieu con la performance de Clark Caminhando de 1963, en la que, con unas tijeras, a partir de una hoja de papel, se obtiene una compleja superficie tridimensional -fig.6-.

"Arriba y abajo, todas las plantas están conectadas por una simple trayectoria, un bulevar interior que expone y relaciona todos los elementos programáticos donde el visitante se convierte en un flâneur baudeleriano, inspeccionando y siendo seducido por un mundo de libros e información, por un escenario urbano" (Koolhaas, 1997: 1320).
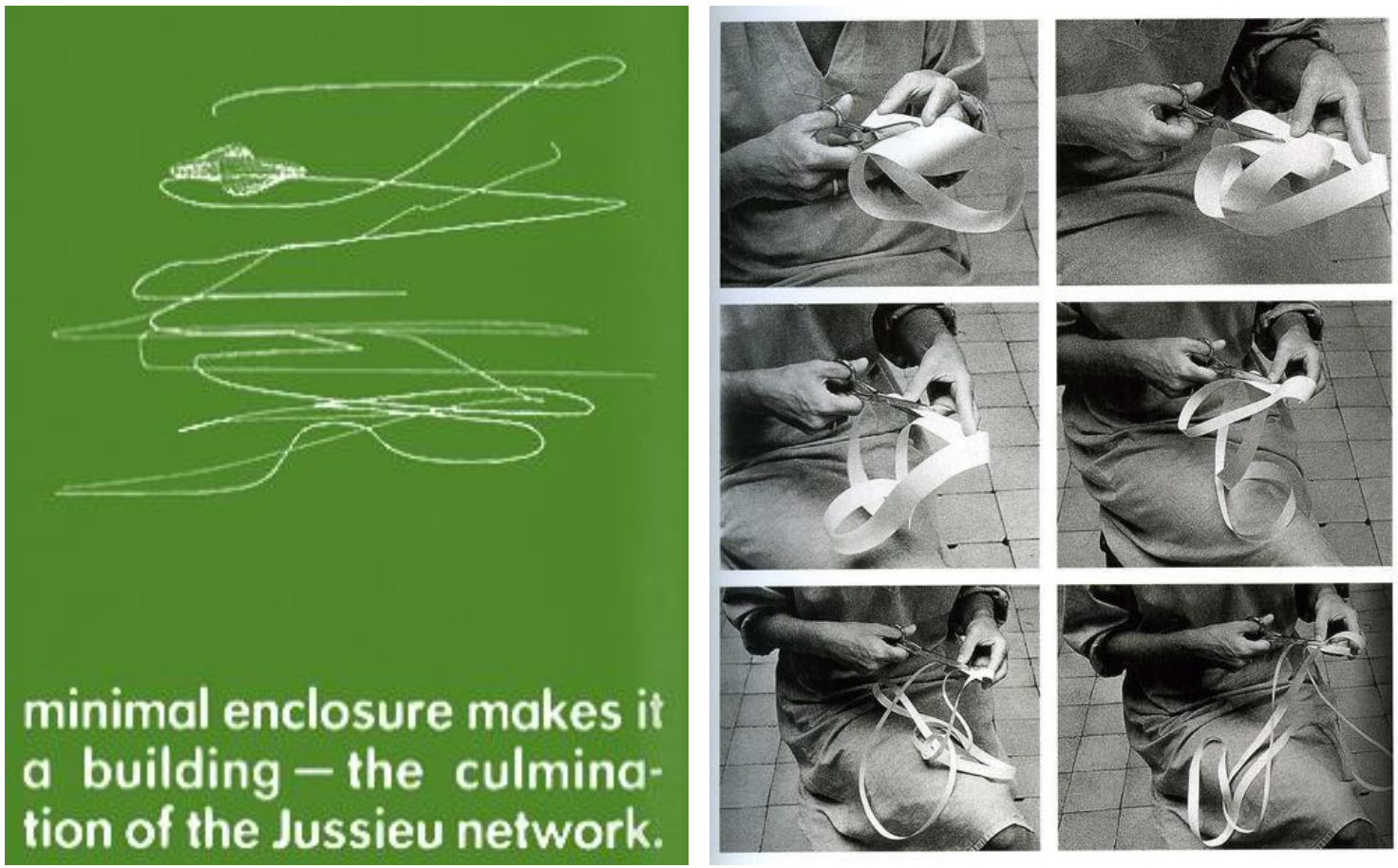

FIG. 6. OMA. S, M, L, XL. Esquema conceptual del deambular por el edificio de las Bibliotecas de Jussieu y la performance de Lygia Clark. Caminhando de 1963.

\section{PLIEGUES. Experimentando con el espacio y el tiempo}

Estos ejemplos, anticipan ya reflexiones sobre la alteración del binomio espacio-tiempo en el que Koolhaas encuentra un territorio fecundo que duda del espacio euclídeo y abre nuevos campos de experimentación sobre lo múltiple, lo divergente, lo simultáneo y lo imposible. De este modo encontraremos una vinculación palpable entre la investigación de Koolhaas y trabajos como los de Gilles Deleuze, sobre el pliegue, en los que reflexiona de cómo este posibilita encuentros múltiples, condensaciones temporales y otras alteraciones (Gargiani, 2008).

"Lo múltiple no es solo lo que tiene muchas partes, sino lo que está plegado de muchas maneras. A cada piso le corresponde un laberinto (...) si Descartes no ha sabido resolverlos es porque ha buscado el secreto del continuo en trayectos rectilíneos, y el de la libertad en una rectitud del alma, ignorando tanto la inclinación del alma como la curvatura de la materia." (Deleuze, 1989: 16) 
Koolhaas había documentado ya en Delirious New York sus descubrimientos sobre las atracciones lúdicas de Coney Island como un laboratorio real de experimentación sobre el espacio y el tiempo: desafíos para el racional entendimiento del movimiento y del espacio, poniendo en duda las habituales categorías de acontecimientos: "lo que está más alejado de la razón, lo que más se ríe de las leyes de la gravitación, es lo que más entusiasma a las multitudes de Coney Island". (Koolhaas, 1978: 34)

Dichos descubrimientos quedan codificados años más tarde en la publicación Content (Koolhaas, 2004), elevándolos a la categoría de inventos. Plantea de modo irónico unas patentes de modernización universal. Es significativo que cinco de dichas patentes se refieran a procesos que tienen que ver con el recorrido arquitectónico vinculado a la condensación espaciotemporal.

En la primera de ellas que denomina Loop-trick: el truco del lazo; define el efecto provocado por la incorporación de una conexión en equis entre dos plantas consecutivas: "introduciendo una $x$ de intersección de plantas en un edificio de dos plantas, se crea una superficie continua que destruye el estatus de la planta individual, se elimina la noción de arriba y abajo" (Koolhaas, 2004: 76). Diríamos nosotros también que se produce un pliegue, una condensación temporal del recorrido del edificio en la intersección de las dos plantas, ya que se trata un punto determinado del espacio al que se accede en dos tiempos distintos y en virtud de dos localizaciones relacionadas con ámbitos diferentes. Esta deformación espacio-temporal es una de las bases proyectuales de la propuesta definitiva para el edificio del Kunsthal de Roterdam, proyecto construido en 1992. En éste edificio, el recorrido principal se concibe como una espiral ascendente que conecta los distintos niveles y en el que se producen varios efectos de condensación que provocan encuentros entre visitantes de distintas partes del programa, en estancias y situaciones divergentes: interior y exterior, arriba y abajo, realidad y reflejo, movimiento ascendente y movimiento descendente; haciendo del recorrido una experiencia cinética indeterminada que se autoenriquece durante la visita al museo. Nos encontramos aquí con un bucle temporal similar al que se producía en el Loop the Loop de Coney Island estudiado por Koolhaas (Koolhaas, 1978). Resulta significativa la frase que sobreescribe OMA sobre la foto de la rampa de acceso al edificio incluida en $S, M, L, X L$ aludiendo de modo inequivoco a la desorientacion provocada por una hipotética alteracion espacio-temporal: "Bien, no estuvimos aquí ayer por la noche. Ahora, ¿qué hicimos ayer por la noche?: redescubriendo la rampa que usaste para entrar" (Koolhaas, 1997: 430) -fig. 7-
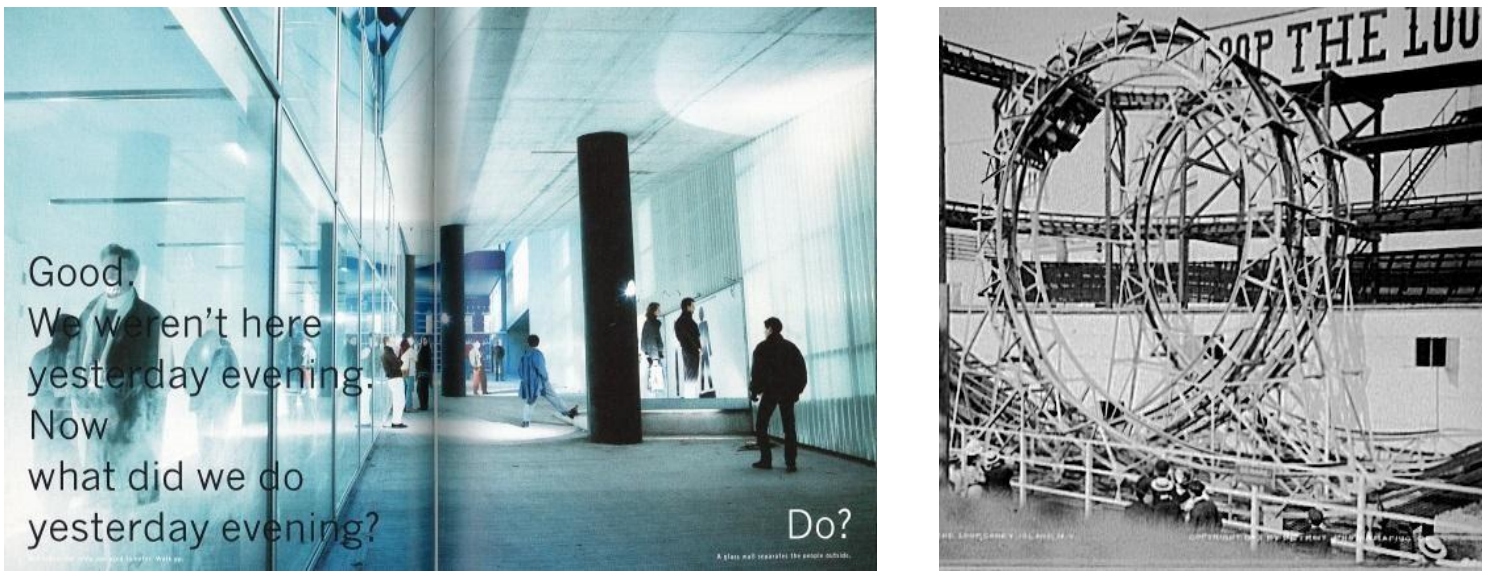

FIG. 7. OMA. Kunsthal de Rotterdam. 1995 y el bucle de circulaciones Loop the Loop, atracción de Coney Island. 1901-1910. 
En el ejemplo del Kunsthal el pliegue en el espacio-tiempo posibilita la condensación en un punto espacial determinado de dos realidades temporales distintas. La novena patente incluida en Content: Everywhere and Nowhere, define exactamente lo contrario: "sistema para transformar un dispositivo de transporte en una habitación para crear una casa cambiante" (Koolhaas, 2004: 81). El ejemplo que propone OMA como demostración del mecanismo, traducido como en todos los sitios y en ninguno, es el proyecto de la casa en Fiorac, cerca de Burdeos, proyecto de 19941998, en el cual transforma un ascensor de comunicación vertical entre las plantas en una plataforma-habitación que comunica todos los niveles. Como comentábamos, interesa el extremo en el que el desplazamiento de una parte de la edificación y su encaje ulterior con los distintos ámbitos de la vivienda posibilita una suerte de teletransportación, un viaje en el espacio sin que el espectador altere su posición (circule) en térm inos relativos - fig. 8-.
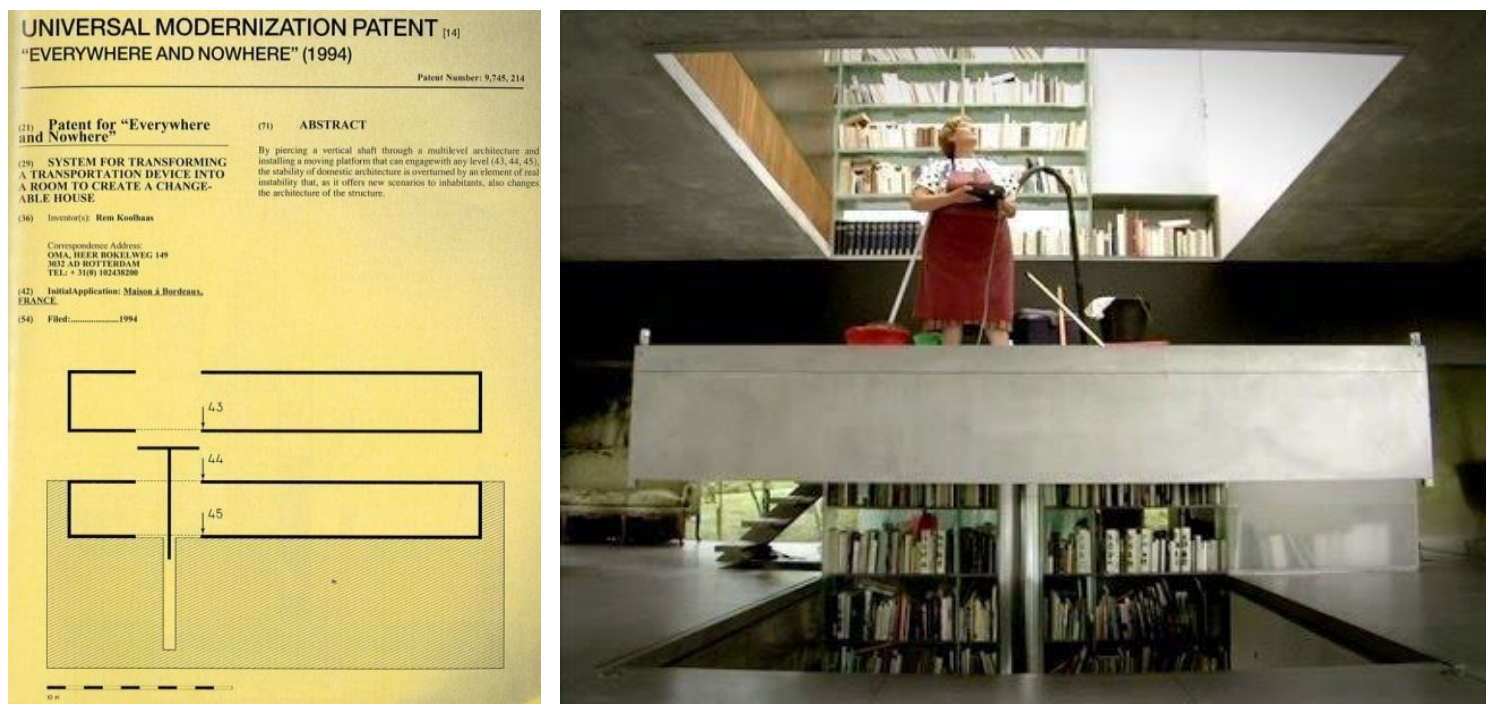

FIG. 8. OMA. Universal Modernization Patent. Incluida en Content. Everywhere and Nowhere. 2004. Junto a la plataforma-habitación de la Casa en Fiorac. Burdeos. 1994-1998.

\section{TRAJECTORIES. La deriva como premisa de proyecto.}

Todos estos ejemplos certifican una evolución del concepto de recorrido en los edificios de OMA que avanzan desde la deambulación libre, a las trajectories preconcebidas como parte integrante del proyecto. Estos recorridos prederminados participan también de los fundamentos surrealistas y situacionistas de deambulación automática, imprevisible y fraccionada, pero no sólo se encuentran definidos ya en la concepción del proyecto, sino que además son un elemento estructurante imprescindible en el mismo. Uno de los primeros proyectos donde se pone de manifiesto el concepto de trajectory implementado a priori por Koolhaas, combinado aún con ciertos grados de libertad en las circulaciones, es el proyecto del ZKM Zentrum für Kunst de Karlsruhe, de 1990. 
Se trata de un gran volumen prismático formalizado por cuatro planos de cerramiento con misión estructural y que incluyen el programa secundario. Todo el vacío central incorpora los espacios principales en varios niveles apilados. Aunque formalmente pudiera recordar al cubo de Jussieu, el funcionamiento de la pieza prismática es diametralmente opuesto: Koolhaas inserta un laberinto dinámico determinado para recorrer todo el complejo en sentido ascendente finalizando en la gran terraza panorámica. La ascensión se aparta de la consideración de un itinerario racional, es cambiante en cada nivel, no sigue ninguna directriz ni vertical ni horizontal, sencillamente va cosiendo las distintas partes del programa, dejando limitada la p osibilidad de deriva automática y libre del visitante - fig. 9-.

"El sistema de circulación pública serpentea alrededor del corazón invadiéndolo en momentos estratégicos en un despliegue continuo de las actividades del Centro" (OMA, 2021).
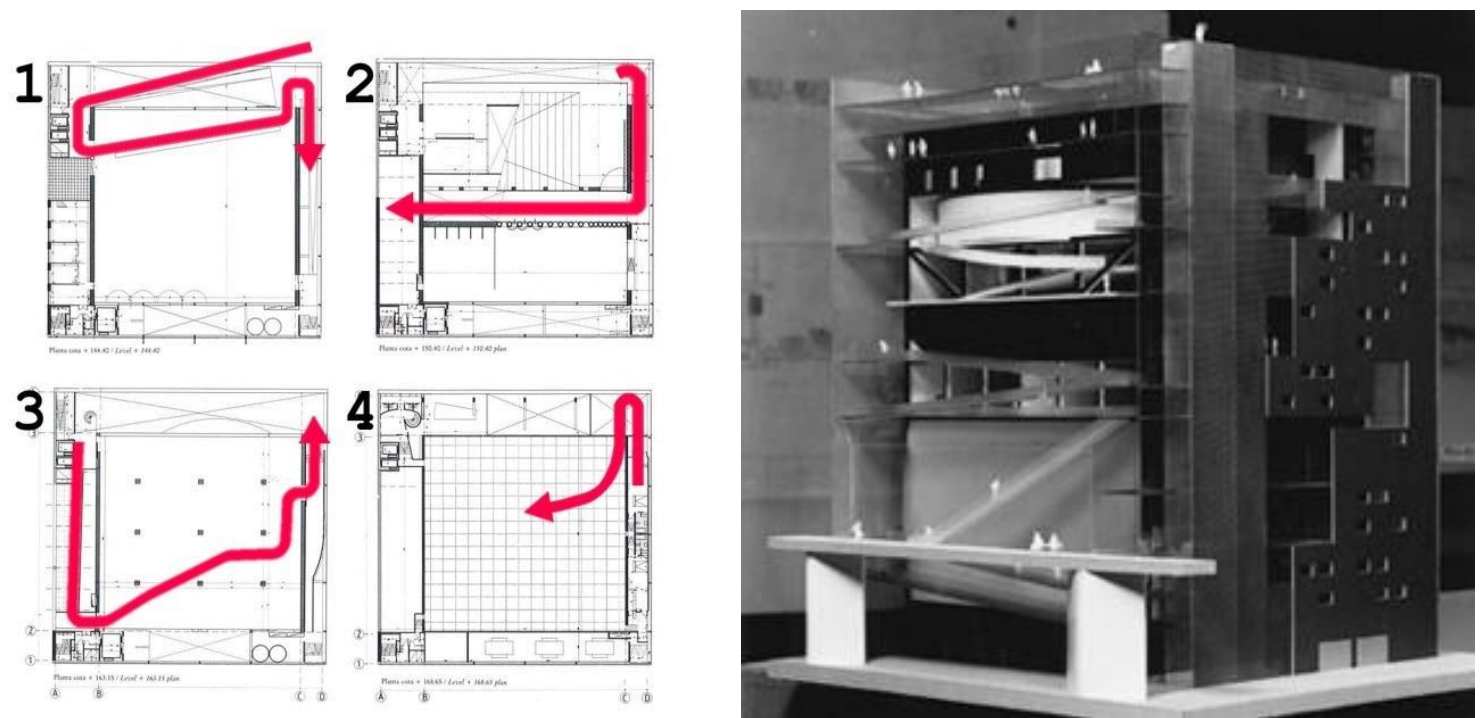

en un hormiguero, se aprecia la complejidad del recorrido ascendente.

La maqueta conceptual de la propuesta para la ampliación del Whitney Museum de Nueva York de 2001 no deja lugar a dudas de que la trajectory es uno de los aspectos fundamentales de la concepción del proyecto. OMA anexa al edificio de Marcel Breuer un gigantesco poliedro irregular, una protuberancia daliniana, que surge sobre los edificios existentes. En este caso, se propone una disposición programática no habitual, alterando la gradación funcional de espacios principales y servidores, e insertando entre los principales ámbitos de exposición, "las más prosaicas de las partes de un museo: zonas comerciales, circulaciones" (Koolhaas, 2004: 217), comunicadas entre sí por la azarosa circulación interior, fundamentada en escaleras mecánicas, que lleva hasta la planta superior. 
Otro ejemplo de una trajectory como punto de partida de la idea del proyecto lo encontramos en el edificio para la embajada de Holanda en Berlín, construido de 2004. OMA organiza el programa en dos volúmenes separados por un vacío, símbolo de los huecos dejados por los bombardeos de la segunda guerra mundial. La configuración platónica y perfecta del cubo que constituye la pieza fundamental del complejo está completamente alterada por la inserción de un recorrido lineal de tortuoso ascenso hasta la cubierta. Se trata de una versión todavía más radical de trajectory que la del edificio del ZKM o la del Whitney, pues en este caso tiene una correspondencia arquitectónica biunívoca con un espacio absolutamente definido y cerrado.

Las maquetas de proyecto evidencian el interés de OMA por la materialización formal de la trajectory por encima del de los espacios que ésta conecta - fig. 10-. La regularidad inicial de la fachada del cubo de cristal y planchas de aluminio, por otra parte, también se ve perturbada cuando el camino interior, como un gusano en el interior de una fruta, roza el exterior, haciéndose visible y proporcionando vistas estratégicas del entorno (OMA, 2021).

En el exterior, la presencia del recorrido se materializa también constructivamente: la trajectory a veces se retranquea y a veces permanece en voladizo, pero siempre se significa interrumpiendo la geometría cartesiana del cubo al que penetra.

“(...) un cubo en el que se disponen las oficinas y un muro, de la misma altura que el cubo (...), semi opaco que rodea el cubo por dos lados, donde se encuentra la vivienda del embajador entre los que surge un patio interior, protegido. Cuatro puentes peatonales atraviesan el patio y vinculan el cubo con el muro en diferentes niveles" (OMA, 2021).
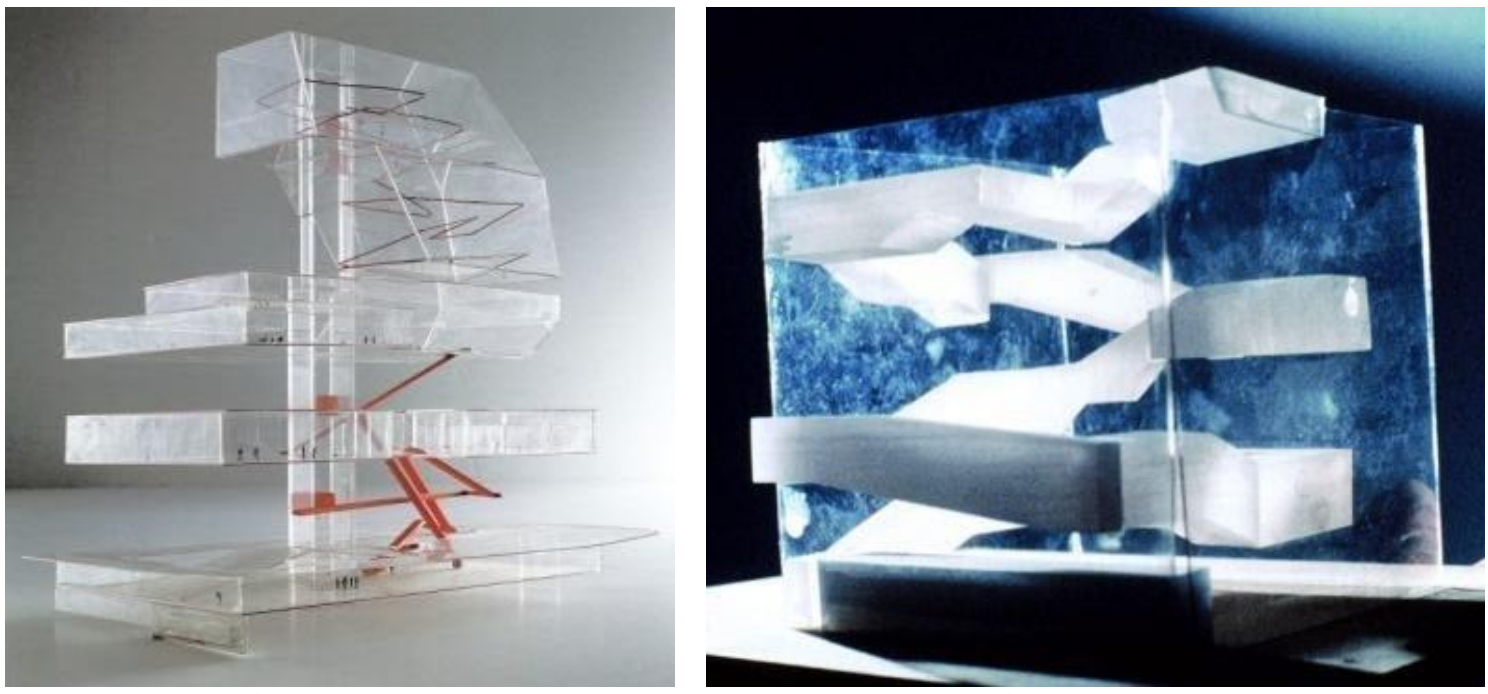

FIG. 10. OMA. ampliación del Whitney Museum de Nueva York y Embajada de Holanda en Berlín. Maquetas conceptuales de la trajectories.

\section{CONCLUSIÓN.}

Es importante señalar que, el presente texto, se inserta en un contexto más amplio de investigación del autor de estas líneas que centra su objetivo en la constante búsqueda de Rem Koolhaas de mecanismos creativos vinculados a lo "no razonable" (Arias, 2020a). Esta vía, le ha permitido al holandés, no solo configurar una obra arquitectónica única, sino posicionarse como uno de los arquitectos más influyentes a partir del último cuarto del pasado siglo XX. Entre estas técnicas destaca sobre otras la utilización de procedimientos "automáticos" entre los que se 
encuadra su particular entendimiento de las circulaciones en los edificios, tratado aquí, pero que le permite, entre otras cosas, construir su teoría de ciudad justificando el automatismo del manhattanismo como doctrina metodológica o también experimentar con el crecimiento volumétrico automático en sus propuestas más notables: edificios incómodos y controvertidos con los que OMA pretende, como hicieran los del grupo de Breton décadas antes, mantener provocativamente la atención en su producción arquitectónica actual (Arias, 2020b).

Con respecto a la circulación, consideramos que este texto demuestra el interés perenne del arquitecto holandés por investigar modos divergentes de transitar la arquitectura que ponen en crisis algunas características frecuentes orientadas casi siempre a facilitar la misma, pero que en ocasiones conducen inexorablemente a la estereotipación de la experiencia del recorrido.

Resuelta muy interesante reflexionar sobre cómo el recorrido arquitectónico que en otros autores se encuentra supeditado a distintos condicionantes del proyecto, se conciba en la mayor parte de las propuestas de Koolhaas como la idea generadora principal. Ese interés aumenta si consideramos que esta idea de recorrido se encuentra apuntalada frecuentemente por mecanismos que tienen que ver con lo poético, lo casual o lo irracional: frente a jerarquía, claridad o eficiencia, nos propone deriva, asombro y emoción. Rem Koolhaas consigue transformar la experiencia urbana del deambular errático en arquitectura; destilar la energía de esa dèrive para organizar el espacio y producir materialidad. 


\section{Referencias}

ARIAS, Javier. "Erotomanía: La actividad paranoico-crítica ejercida por Rem Koolhaas sobre Le Corbusier y Mies van der Rohe" RITA. Revista Indexada de Textos académicos. no 14. Madrid: Red Fundamentos. 2020a. pp. 124-131. https://doi.org/10.24192/2386-7027(2020)(v14)(08)

ARIAS, Javier. "Fuera de Contexto. Rem Koolhaas y el objet trouvé" en Revista de Arquitectura. no 25-38. Santiago de Chile: Universidad de chile. 2020b. pp. 45-52. https://dearquitectura.uchile.cl/index.php/RA/article/view/56983

ARIAS, Javier. La construcción del sueño. Poética surrealista en la arquitectura de Rem Koolhaas. Tesis doctoral. Universidad de Valladolid. 2016 https://doi.org/10.35376/10324/33071

BAUDELAIRE, Charles. Poemas en prosa. Rosario: El Cid Editor, 2003.

BASAR, Shumon. The world of Madelon Vriesendorp, Londres: AA publications, 2008.

BENJAMIN, Walter, “El surrealismo, la última instantánea de la inteligencia europea, 1929”, en Imaginación y Sociedad. Iluminaciones I. Madrid: Editorial Taurus, 1980. pp. 41.

BRETON, André. Manifiestos del surrealismo. Madrid: Guadarrama, 1969.

CARERI, Francesco. Walkscapes. El andar como práctica estética. Barcelona: Gustavo Gili, 2015.

CONSTANT. La Nueva Babilonia. Barcelona: Gustavo Gili, 2011.

CORTÉS, Juan Antonio. “Delirio y Más”, en El Croquis, n. 131/132, 2006. pp. 4-28.

DEBORD, Guy. "Teoría de la Deriva (1958)" en el \# 2 de Internationale Situationniste. Traducción extraída de Internacional Situacionista, vol. I: La realización del arte, Madrid: Literatura Gris, 1999.

del VALLE, Raul. La herencia de Le Corbusier en la arquitectura de Rem Koolhaas. Tesis doctoral. Universidad Politécnica de Madrid. 2006. https://doi.org/10.20868/UPM.thesis.44464

DELEUZE, Gilles. El Pliegue. Leibniz y el Barroco. Barcelona: Paidós Ibérica, 1989.

DUCATEZ, Vicent. "El jardín del placer de OMA" en Revista Bitácora Urbano Territorial 9, no 1. Colombia: Revistas Unal, 2005. pp. 8-16. https://revistas.unal.edu.co/index.php/bitacora/article/view/18731

GARCíA, Carlos. Atlas de Exodus. Tesis doctoral. Universidad Politécnica de Madrid. 2014. https://oa.upm.es/30888/

GARGIANI, Roberto. Rem Koolhaas/OMA. The construction of Merveilles. Lausana: EPFL Press. 2008.

KOOLHAAS, Rem. Delirious New York. Oxford: Oxford University Press, 1978.

KOOLHAAS, Rem, “Dos Bibliotecas en Jussieu”, en El Croquis no 79. 1996. pp.124-141.

KOOLHAAS, Rem and Mau, Bruce. S, M, L, XL. Nueva York. Monacelli Press. 1997.

KOOLHAAS, Rem, ed. Content. AMOMA/ REM KOOLHAAS/ \&\&\& Simon Brown \& Jon Link. Köln: Taschen, 2004.

MOSQUERA, Joaquín. Conectividad urbana en Rem Koolhaas. Megaestructura, calle elevada e infraestructura de comunicación. Tesis doctoral. Universidad politécnica de Madrid. 2016. https://doi.org/10.20868/UPM.thesis.44464

OMA, 2021. Página web de OMA. Accesible en http://www.oma.eu.

QUETGLÁS, Josep. Promenade architecturale. En: Web Architecture Magazine. 05. Artículo en línea. Disponible en: http://www.arranz.net/web.arch-mag.com/5/homeless/05s.html

WILEY Danielle. "A Walk About Rome: Tactics for Mapping the Urban Periphery" En Architectural Theory Review, no15:1, 2010. pp. 9-29. https://doi.org/10.1080/13264821003629220

YARIMBAŞ. Duygu. "Experiencing city by walking: Communication elements" en A|Z ITU Journal of Faculty of Architecture. Vol 15, no 2. July 2018. pp. 19-30. https://doi.org/10.5505/itujfa.2018.87360

ZAERA, Alejandro. "Conceptual Evolution of the work of Rem Koolhaas" en Rem Koolhaas: Projectes Urbans (19851990). Barcelona: Colegio de Arquitectos de Cataluña, 1990. 


\section{Bio}

Doctor Arquitecto. Profesor Ayudante Doctor del Departamento de Construcciones Arquitectónicas de la ETSA de Valladolid.

Doctor of Architecture. Assistant Professor of the Department of Architectural Constructions of the ETSA of Valladolid. 